\title{
Chirped polymer optical fiber Bragg grating sensors
}

\author{
Carlos A. F. Marques*a,b, L. Pereira ${ }^{\mathrm{b}}$, P. Antunes ${ }^{\mathrm{b}}$, P. Mergo ${ }^{\mathrm{c}}$, D. J. Webb ${ }^{\mathrm{d}}$, J. L. Pinto ${ }^{\mathrm{b}}$, P. André \\ anstituto de Telecomunicações, Campus de Santiago, 3810-193 Aveiro, Portugal \\ bUniversity of Aveiro, Physics Department, Campus de Santiago, 3810-193 Aveiro, Portugal \\ ${ }^{c}$ Laboratory of Optical Fibers Technology, Maria Curie Sklodowska University, Lublin, Poland \\ ${ }^{\mathrm{d}}$ Aston Institute of Photonic Technologies, Aston University, B4 7ET Birmingham, UK \\ ${ }^{\mathrm{e}}$ Instituto de Telecomunicações and Department of Electrical and Computer Engineering, Instituto \\ Superior Técnico, University of Lisbon, 1049-001 Lisbon, Portugal \\ *E-mail: carlos.marques@ua.pt
}

\begin{abstract}
We report chirped fiber Bragg gratings (CFBGs) photo-inscribed in undoped PMMA polymer optical fibre (POF) for the first time. The chirped polymer optical fiber Bragg gratings (CPOFBGs) were inscribed using an UV KrF excimer laser operating at $248 \mathrm{~nm}$. The rectangular gauss laser beam was expanded to $25 \mathrm{~mm}$ in horizontal direction along the fiber core by a cylindrical lens, giving a total of $25 \mathrm{~mm}$ grating length. A $25 \mathrm{~mm}$ long chirped phase mask chosen for $1550 \mathrm{~nm}$ grating inscription was used. The laser frequency was $1 \mathrm{~Hz}$ with an energy of $5 \mathrm{~mJ}$ per exposure, exposing few pulses for each grating inscription. The reflection amplitude spectrum evolution of a CPOFBG is investigated as a function of the applied strain and temperature. Also, some results regarding to group delay are collected and discussed. These results pave the way to further developments in different fields, where POFs could present some advantages preferably replacing their silica counterparts.
\end{abstract}

Keywords: Fiber Bragg gratings; Polymer optical fiber sensors; chirp, sensing.

\section{INTRODUCTION}

FBGs in POF (POFBGs) are claimed to offer an interesting alternative to silica FBGs for sensing applications [1-4]. There are a several distinct types of FBG structures such as: the uniform Bragg grating characterized by a constant grating pitch (spacing between grating planes), the tilted FBG (TFBG) which has the grating planes tilted with respect to the fiber axis, and the chirped Bragg grating (CFBG) that has a non-periodic pitch, displaying a linear variation in the grating pitch, called a chirp [5,6]. CFBGs or also called aperiodic Bragg gratings are known in the literature for gratings with chirp and generally refer to gratings in which the resonance condition varies along its length. If the variation of the periodicity is small, it can be considered locally uniform. Because of this, each part of the grating will reflect different wavelengths without affecting each other [7]. The most common aperiodic FBGs are gratings with variable period along its longitudinal extension. The sensing element behaves as a layer of FBGs, in which different Bragg wavelengths are encoded along the fiber axis $\mathrm{z}$ [6]. The modulation period $\Lambda(\mathrm{z})$ follows a linear profile $\left.\Lambda(z)=\Lambda_{0}+k z\right)$, for $0<\mathrm{z}<\mathrm{L}$, where $\mathrm{L}$ is the grating length; $\mathrm{k}$ is the chirp coefficient, which defines the increase of refractive index period per unit of length. Of course, the period of an aperiodic FBG can be expressed by a polynomial of $\mathrm{N}$ degree given as $\Lambda(\mathrm{z})=\Lambda 0+\Lambda 1 \mathrm{z}+\ldots+\Lambda \mathrm{N} \mathrm{zN}$, which is important for optical communications field. The aperiodicity has effect in two properties of Bragg gratings: the bandwidth and group delay. The group delay characteristics of the grating are different from conventional gratings. Since different wavelengths are reflected in different positions on the fiber, it causes a group delay dependent on the wavelength. This kind of FBGs and analysis are used in optical communications to compensate the dispersion in different situations or others applications [14,15]. For sensing applications, there are many works using silica CFBGs for refractive index and strain measurements, biomedical, splits and transverse cracks in structural health monitoring (SHM) by local pressure analysis, or liquid level monitoring $[10,11]$. For the aforementioned applications, POFs bring additional benefits, such as robustness, cost-effectiveness, ease of installation and improved biocompatibility. Also, photo-inscription in POFs has matured to such a degree that it now becomes feasible to investigate CPOFBGs fabrication, which requires more efficiency than uniform FBGs. 


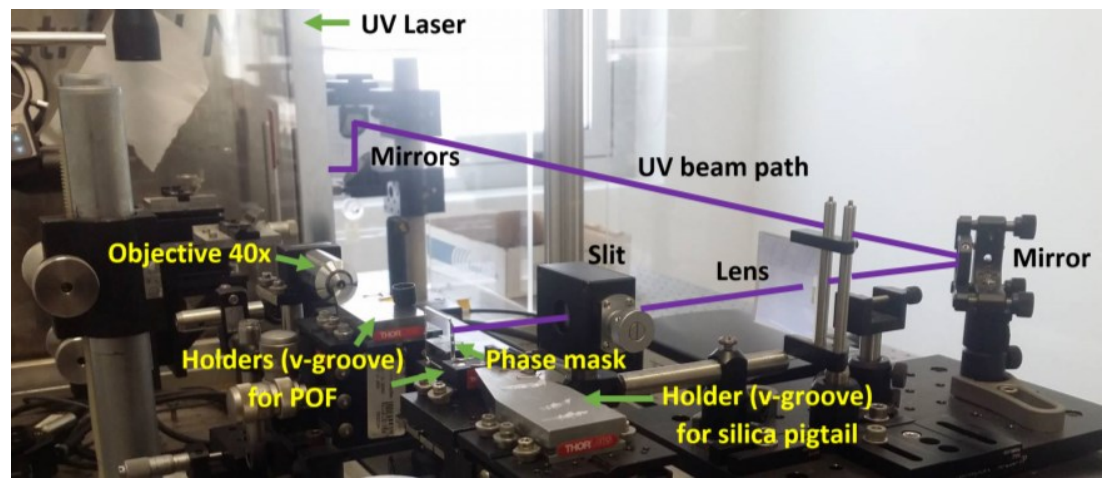

Proc. of SPIE Vol. 10232 102320N-2 

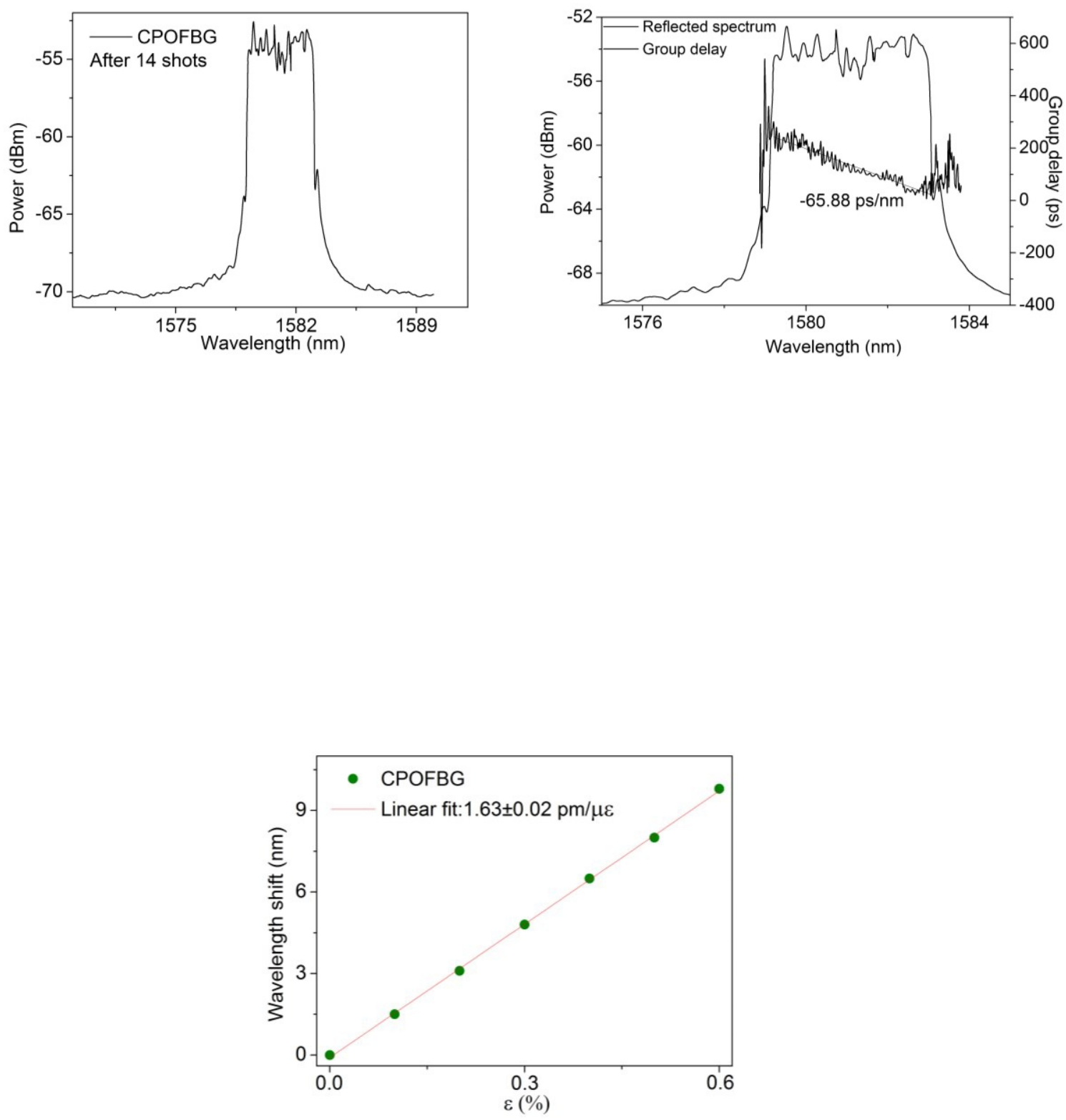

Proc. of SPIE Vol. 10232 102320N-3 

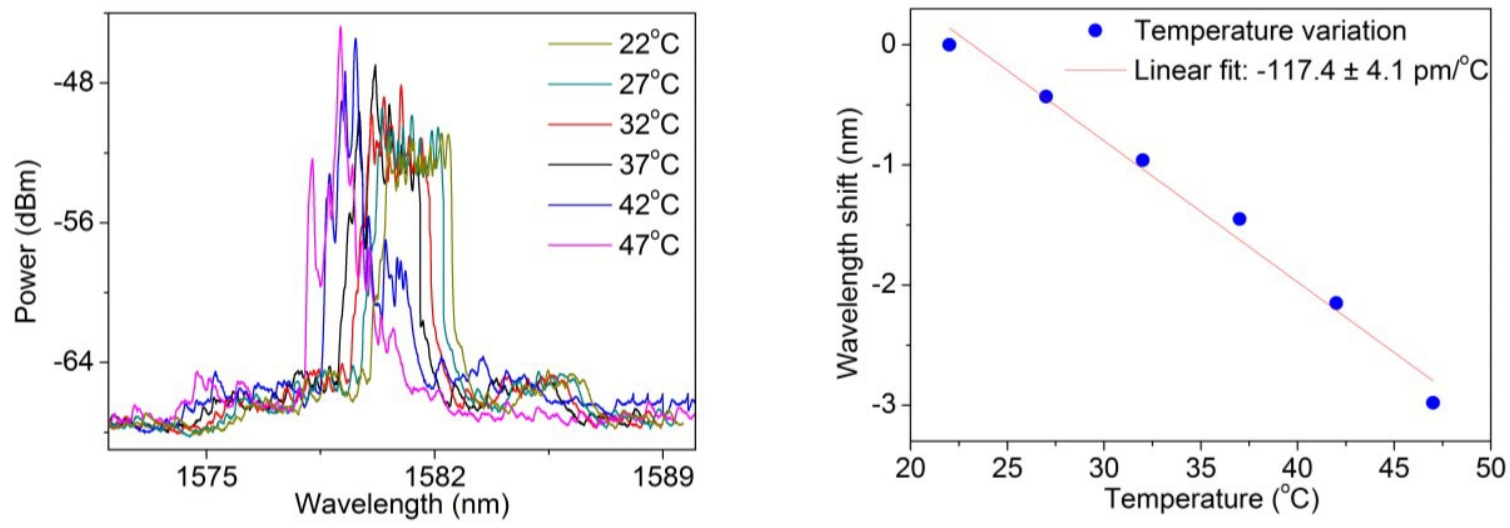

Proc. of SPIE Vol. 10232 102320N-4 
4. D. J. Webb, "Fibre Bragg grating sensors in polymer optical fibres," Meas. Sci. Technol. 26, 092004 (2015).

5. R. Kashyap, Fiber Bragg Grating, Academic Press, New York (1999).

6. T. Erdogan, "Fiber grating spectra," J. Lightwave Technol. 15, 1277 (1997).

7. K. C. Byron, K. Sugden, T. Bricheno, I. Bennion, "Fabrication of chirped Bragg gratings in photosensitive fibre", Elect. Letters 29, 1659 (1993).

8. B. J. Eggleton, A. Ahuja, P. S. Westbrook, J. A. Rogers, P. Kuo, T. N. Nielsen, B. Mikkelsen, "Integrated Tunable Fiber Gratings for Dispersion Management in High-Bit Rate Systems," J. Lightwave Technol. 18, 1418, (2000).

9. M. E. Fermann, K. Sugden, I. Bennion, "High-Power Soliton Fiber Laser Based on Pulse Width Control with Chirped Fiber Bragg Gratings," Opt. Lett. 20, 172 (1995).

10. A. Sun, Z. Wu, "A Hybrid LPG/CFBG for Highly Sensitive Refractive Index Measurements", Sensors 12, 7318 (2012).

11. D. Tosi, E. G. Macchi, M. Gallati, G. Braschi, A. Cigada, S. Rossi, G. Leen, E. Lewis, "Fiber-optic chirped FBG for distributed thermal monitoring of ex-vivo radiofrequency ablation of liver," Biomed. Opt. Express 5, 1799 (2014).

12. C. A. F. Marques, A. Pospori, G. Demirci, O. Çetinkaya, B. Gawdzik, P. Antunes, P. Mergo, O. Bang, P. André, D. J. Webb, "Fast Bragg gratings inscription using PMMA polymer optical fibers: impact of thermal pre-treatment on preforms", in 25th International Conference on Plastic Optical Fibres - POF 2016, ISBN: 978185449408 5, (2016).

13. C. A. F. Marques, Fiber-Optic Components for Optical Communications and Sensing, PhD Thesis, Universidade de Aveiro, (2013).

14. O. Frazão, M. Melo, P. V. S. Marques, J. L. Santos, "Chirped Bragg grating fabricated in fused fibre taper for straintemperature discrimination," Meas. Sci. Technol. 16, 984 (2005).

15. H. Liu, H. Liu, G.-D. Peng, T. W. Whitbread, "Tunable dispersion using linearly chirped polymer optical fiber Bragg gratings with fixed center wavelength," IEEE Photon. Technol. Lett. 17, 411 (2005). 